\title{
Downlink Power Control and Base Station Assignment
}

\author{
Farrokh Rashid-Farrokhi, Student Member, IEEE, K. J. Ray Liu, Member, IEEE, \\ and Leandros Tassiulas, Member, IEEE
}

\begin{abstract}
The joint power control and base station assignment for downlink is considered in this paper. Based on the results and observations of previous work we present an algorithm that finds a feasible joint base assignment and transmission power vector in the downlink. We show that under some conditions the algorithm also minimizes the sum of downlink powers. Interestingly, there is not always a Pareto optimal power vector for the joint problem in the downlink as is the case in the uplink. This fact is demonstrated through a counterexample.
\end{abstract}

\section{INTRODUCTION}

$\mathbf{T}$ HE OBJECTIVE of power control in wireless networks is to minimize transmitted powers subject to maintaining the link quality by keeping the signal-to-interference ratio (SIR) above a threshold called minimum protection ratio. The power allocation and base station assignment can be integrated to attain higher capacity and achieve smaller allocated powers. In a network with power control capability, as the mobiles move or new calls arrive, the reassignment of mobiles to base stations helps to find a feasible power allocation and provides a framework for handoff.

In [1]-[3] the combined base station assignment and power allocation was proposed for uplink. In those papers, algorithms were proposed to achieve the optimal base assignment and power allocation such that the mobile powers are minimal among all feasible assignments. In [2] it has been observed that the base station assignment in the downlink cannot be done using the algorithms proposed in [1]-[3]. Based on the simple observation in [4] that for fixed assignment the feasibility of uplink and downlink are equivalent, in this letter we consider the joint power control and base station assignment for downlink. It is interesting to note that unlike the fixed assignment, in the joint problem, the uplink and downlink are substantially different. While in the uplink there is a Pareto optimal solution for the power control and base station assignment, this is not the case for the downlink. We will demonstrate this fact through a counterexample. Note that when there is a Pareto optimal solution the globally optimal solution is achieved when each user attempts to optimize his own power while in the other case the issue of power allocation

Manuscript received March 5, 1997. This work was supported in part by the National Science Foundation under NYI Award MIP9457397 and by the National Science Foundation under the NSF CAREER Award. The associate editor coordinating the review of this letter and approving it for publication was Dr. B. Vojcic.

The authors are with the Electrical Engineering Department and Institute for Systems Research, University of Maryland at College Park, College Park, MD 20742 USA.

Publisher Item Identifier S 1089-7798(97(05910-3. arises. Regarding the joint problem in the downlink we will show that the sequence of base station assignment for the joint uplink power control and base station assignment converges to a feasible assignment for the downlink. We use the same iterations to update the downlink power. Despite the fact that the downlink power update is not a standard function [2], we will prove that this algorithm converges to a feasible downlink power allocation where there exists any. We will also show that when the thermal noise power at all receivers are equal, the proposed algorithm also minimizes the sum of downlink powers among all feasible assignments.

\section{UPLINK POWER CONTROL AND BASE STATION ASSignMENT}

The aim of power control is to minimize transmitted power such that the SIR at each link (denoted by $\Gamma_{i}$ ) is no less than a threshold $\gamma_{i}$. That is,

$$
\Gamma_{i}=\frac{G_{i i} P_{i}}{\sum_{j \neq i} G_{j i} P_{j}+N_{i}} \geq \gamma_{i}, \quad i=1, \cdots, M
$$

where $M$ is the number of mobiles, $G_{j i}$ is the link gain between mobile $j$ and base $i, P_{i}$ is the $i$ th mobile power, and $N_{i}$ is the noise power at the $i$ th base station. The above constraint in matrix form is given by $\boldsymbol{P} \geq \boldsymbol{D F P}+u$, where $\boldsymbol{P}=\left[P_{1}, \cdots, P_{M}\right]^{T}, \boldsymbol{D}=\operatorname{diag}\left\{\gamma_{1} / G_{11}, \cdots, \gamma_{M} / G_{M M}\right\}, \boldsymbol{u}$ is an element-wise positive vector whose elements are defined as $u_{i}=\gamma_{i} N_{i} / G_{i i}$, and

$$
[\boldsymbol{F}]_{i j}= \begin{cases}0 & \text { if } j=i \\ G_{j i} & \text { if } j \neq i .\end{cases}
$$

If the spectral radius of $\boldsymbol{D F}$ is less than unity, i.e., $\rho(\boldsymbol{D F})<1$, the network is feasible and the optimal solution to the power control problem is given by $\hat{P}=[I-D F]^{-1} \boldsymbol{u}$. Denote the $i$ th base station power by $\tilde{P}_{i}$ and the noise power at the $i$ th mobile by $\tilde{N}_{i}$. Similar to uplink, it can be shown that if $\rho\left(\boldsymbol{D F} \boldsymbol{F}^{T}\right)<1$, the optimal downlink power is given by $\hat{\tilde{\boldsymbol{P}}}=\left[I-\boldsymbol{D} \boldsymbol{F}^{T}\right]^{-1} \tilde{\boldsymbol{u}}$, where $\tilde{\boldsymbol{P}}=\left[\tilde{P}_{1}, \cdots, \tilde{P}_{M}\right]^{T}$, and $\tilde{u}_{i}=\gamma_{i} \tilde{N}_{i} / G_{i i}$. In [4] it has been shown that the spectral radius of $\boldsymbol{D F}$ and $\boldsymbol{D} \boldsymbol{F}^{T}$ and consequently the feasibility of uplink and downlink power control with fixed assignment are the same.

In the optimal uplink power control and base station assignment, the objective is to determine the assignment of users to base stations which minimizes the allocated mobile powers. Iterative algorithms that compute the optimal joint base station 
and power assignment for the uplink were proposed in [1]-[3]. The power update in this case is given by

$$
P_{i}^{n+1}=\arg \min _{k \in B_{i}}\left\{\sum_{j \neq k} \frac{\gamma_{i} G_{j k}}{G_{i k}} P_{j}^{n}+\frac{\gamma_{i} N_{k}}{G_{i k}}\right\}
$$

where $B_{i}$ is the set of allowable base stations for the $i$ th mobile. A network is feasible if there exists an assignment and power allocation such that the quality constraint is satisfied for each link. In [1]-[3] it has been shown that in a feasible network, the above iteration converges to the optimal power allocation and base station assignment, such that the mobile power is minimal among all feasible base station assignments.

\section{Downlink POWER CONTROL AND BASE STATION ASSIGNMENT}

In [2] it has been observed that the standard methods for base station assignment and power control cannot be applied to find the optimal base station assignment in the downlink, because the interference reduction associated with changing the base station assignment of one user may result in greater interference to other links [2]. In this letter, we will show through a counterexample that there is no Pareto optimal solution for the joint problem in the downlink. Using known results from the joint uplink problem and the downlink-uplink equivalence for the fixed assignment power control problem, we enhance the joint power allocation and base station assignment by the following algorithm. We let the uplink select the base station and at the same time we run one power control iteration for downlink using the same assignment. The algorithm steps at the $n$th iteration are as follows.

\section{Algorithm BUD:}

1) (B) The base station assignment, denoted by $b_{i}^{n}$, is found to minimize the uplink power:

$$
b_{i}^{n}=\arg \min _{k \in B_{i}}\left\{\sum_{j \neq i} \frac{\gamma_{i} G_{j k}}{G_{i k}} P_{j}^{n}+\frac{\gamma_{i} N_{k}}{G_{i k}}\right\} .
$$

2) (U) The uplink power is updated based on the assignment $b_{i}^{n}$ :

$$
P_{i}^{n+1}=\sum_{j \neq i} \frac{\gamma_{i} G_{j b_{i}^{n}}}{G_{i b_{i}^{n}}} P_{j}^{n}+\frac{\gamma_{i} N_{i}}{G_{i b_{i}^{n}}} .
$$

3) (D) The downlink power is updated based on the same assignment $\left(b_{i}^{n}\right)$ :

$$
\tilde{P}_{i}^{n+1}=\sum_{j \neq i} \frac{\gamma_{i} G_{i b_{j}^{n}}}{G_{i b_{i}^{n}}} \tilde{P}_{j}^{n}+\frac{\tilde{N}_{i} \gamma_{i}}{G_{i b_{i}^{n}}} .
$$

Based on the fact that the power update for uplink is a standard function, it has been shown ([2]) that the first two steps of the above algorithm converge to the optimal solution. The downlink power update is not a standard function, and as a result the convergence of the algorithm cannot be shown using the method in [2]. However, using the simple fact that the same assignment is used in the uplink and downlink at each iteration, we will prove the following.
Theorem 1: The downlink power update in Algorithm BUD converges to a feasible power allocation for the downlink.

Proof: In [3] it has been proved that for any starting power allocation there exists an $N \geq 0$ such that for $n \geq N, b_{i}^{n}=\hat{b}_{i}$. Consider the positive vectors $\hat{\boldsymbol{u}}$ and $\hat{\tilde{\boldsymbol{u}}}$ whose elements are defined by $\hat{u}_{i}=\left(\gamma_{i} N_{i}\right) / G_{\widehat{i b}_{i}}$ and $\hat{\tilde{u}}_{i}=$ $\left(\gamma_{i} \tilde{N}_{i}\right) / G_{\widehat{i b}_{i}}$. Define $\hat{D}=\operatorname{diag}\left\{\gamma_{1} / G_{\widehat{b}_{1}}, \cdots, \gamma_{M} / G_{M \hat{b}_{M}}\right\}$, and $\hat{\boldsymbol{F}}=\left\{\hat{F}_{i j}\right\}$

$$
\hat{F}_{i j}= \begin{cases}0 & \text { if } j=i \\ G_{\widehat{b}_{i}} & \text { if } j \neq i .\end{cases}
$$

For $n>N$ the power control iteration for uplink and downlink can be written in matrix form as $P^{n}=\hat{D} \hat{\boldsymbol{F}} \boldsymbol{P}^{n-1}+\hat{\boldsymbol{u}}$ and $\tilde{\boldsymbol{P}}^{n}=\hat{\boldsymbol{D}} \hat{\boldsymbol{F}}^{T} \tilde{\boldsymbol{P}}^{n-1}+\hat{\tilde{\boldsymbol{u}}}$, where $\boldsymbol{P}^{n}=\left[P_{1}^{n}, \cdots, P_{M}^{n}\right]^{T}$ and $\tilde{P}^{n}=\left[\tilde{P}_{M}^{n}, \cdots, \tilde{P}_{M}^{n}\right]^{T}$. If the spectral radius of $\hat{\boldsymbol{D}} \boldsymbol{F}$ is less than unity the above iteration converges to [5] $\hat{\boldsymbol{P}}=[I-$ $\hat{D} \hat{\boldsymbol{F}}]^{-1} \hat{\boldsymbol{u}}$. Since $\rho(\hat{\boldsymbol{D}} \hat{\boldsymbol{F}})=\rho\left(\hat{\boldsymbol{D}} \hat{\boldsymbol{F}}^{T}\right)$ the optimal assignment for uplink is feasible for downlink as well, and the downlink power iterations converge to $\hat{\tilde{\boldsymbol{P}}}=\left[I-\hat{\boldsymbol{D}} \hat{\boldsymbol{F}}^{T}\right]^{-1} \hat{\hat{\boldsymbol{u}}}$.

Note that the thermal noise power solely depends on the bandwidth and noise figure of a receiver, which is the same for all receivers. That is, $N_{i}$ are the same for all base stations and $\tilde{N}_{i}$ are the same for all mobiles. In the following we will show that the sum of base station powers is also minimal among all feasible base station assignments.

Theorem 2: In the case that $N_{i}=N$ and $\tilde{N}_{i}=\tilde{N}$, Algorithm BUD also minimizes $\Sigma_{i} \tilde{P}_{i}$.

Proof: It can be shown that the downlink and uplink power allocations, computed by algorithm BUD, are given by $\hat{\boldsymbol{P}}=N \sum_{k=0}^{\infty}(\hat{\boldsymbol{D}} \hat{\boldsymbol{F}})^{k} \hat{\boldsymbol{D}} \mathbf{1}$, and $\hat{\tilde{\boldsymbol{P}}}=\tilde{N} \sum_{k=0}^{\infty}\left(\hat{\boldsymbol{D}} \hat{\boldsymbol{F}}^{T}\right)^{k} \hat{\boldsymbol{D}} \mathbf{1}$, respectively, where 1 is the all one vector. Here we used the fact that $\hat{\boldsymbol{u}}=N \hat{\boldsymbol{D}} \mathbf{1}$ and $\hat{\tilde{\boldsymbol{u}}}=\hat{N} \hat{\boldsymbol{D}} \mathbf{1}$. The sum of mobile powers (uplink) is given by

$$
\mathbf{1}^{T} \hat{\boldsymbol{P}}=N \sum_{k=0}^{\infty} \mathbf{1}^{T}(\hat{\boldsymbol{D}} \hat{\boldsymbol{F}})^{k} \hat{\boldsymbol{D}} \mathbf{1}
$$

and the sum of base station powers (downlink) is given by

$$
\mathbf{1}^{T} \hat{\tilde{\boldsymbol{P}}}=\tilde{N} \sum_{k=0}^{\infty} \mathbf{1}^{T}\left(\hat{\boldsymbol{D}} \hat{\boldsymbol{F}}^{T}\right)^{k} \hat{\boldsymbol{D}} \mathbf{1} .
$$

Since the sum of base station powers is a scaler, we can transpose the right-hand side of the above equation, i.e.,

$$
\begin{aligned}
\mathbf{1}^{T} \hat{\tilde{\boldsymbol{P}}} & =(\hat{\tilde{\boldsymbol{P}}})^{T} \mathbf{1}=\tilde{N} \sum_{k=0}^{\infty} \mathbf{1}^{T} \hat{\boldsymbol{D}}(\hat{\boldsymbol{F}} \hat{\boldsymbol{D}})^{k} \mathbf{1} \\
& =\frac{\tilde{N}}{N} N \sum_{k=0}^{\infty} \mathbf{1}^{T}(\hat{\boldsymbol{D}} \hat{\boldsymbol{F}})^{k} \hat{\boldsymbol{D}} \mathbf{1}=\frac{\tilde{N}}{N} \mathbf{1}^{T} \hat{\boldsymbol{P}} .
\end{aligned}
$$

The optimal base station assignment and power control achieves the minimal power vector among all feasible assignments, and the sum of uplink and downlink powers are the same up to a constant. Therefore, the sum of powers for downlink is also minimal among all feasible assignments. $\square$

In the following we show that, in general, there is no pointwise optimal solution to the joint power control and base station assignment in the downlink. Consider a downlink 


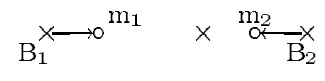

(a)

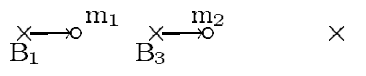

(b)
Fig. 1. Feasible assignments for uplink; base stations are shown by $\times$ and mobiles are shown by $\circ$.

network of two mobiles and three base stations as illustrated in Fig. 1. We consider two assignments of base stations to mobiles. In the first assignment $\left(a_{1}\right), m_{1}$ is assigned to $B_{1}$ and $m_{2}$ is assigned to $B_{2}$. In the second assignment $\left(a_{2}\right) m_{1}$ is assigned to $B_{1}$ and $m_{2}$ is assigned to $B_{3}$. Let the noise power at all mobiles be $\tilde{N}$ and the SNR threshold be $\gamma$. The minimum power allocation which satisfies the link quality is given by

$$
\tilde{P}_{1}^{a_{1}}=\alpha\left(\frac{\gamma G_{12}}{G_{11} G_{22}}+\frac{1}{G_{11}}\right)
$$

and

$$
\tilde{P}_{2}^{a_{1}}=\alpha\left(\frac{\gamma G_{21}}{G_{11} G_{22}}+\frac{1}{G_{22}}\right)
$$

where $\alpha=\gamma \tilde{N} /\left(1-\gamma^{2} G_{12} G_{21} /\left(G_{11} G_{22}\right)\right)$. In Fig. 1(a) $m_{1}$ is closer to its base station. Therefore, $G_{11}>G_{22}$ and $G_{12}<G_{21}$. In this case $\tilde{P}_{1}^{a_{1}}<\tilde{P}_{2}^{a_{1}}$. Similarly, in Fig. 1(b) the optimal power vectors are given by

$$
\tilde{P}_{1}^{a_{2}}=\beta\left(\frac{\gamma G_{13}}{G_{11} G_{23}}+\frac{1}{G_{11}}\right)
$$

and

$$
\tilde{P}_{2}^{a_{2}}=\beta\left(\frac{\gamma G_{21}}{G_{11} G_{23}}+\frac{1}{G_{23}}\right)
$$

where $\beta=\gamma \tilde{N} /\left(1-\gamma^{2} G_{13} G_{21} /\left(G_{11} G_{23}\right)\right)$. In this case since $m_{2}$ is closer to its assigned base station, $G_{11}<G_{23}$ and $G_{13}>G_{21}$, i.e., $\tilde{P}_{2}^{a_{2}}<\tilde{P}_{1}^{a_{2}}$. Also if the location of $B_{3}$ is close enough to $m_{2}$ such that $G_{13} / G_{23}>G_{12} / G_{22}$, then $\tilde{P}_{1}^{a_{1}}<\tilde{P}_{1}^{a_{2}}$. That is, by changing the assignment from Fig. 1(a) to (b), $\tilde{P}_{1}$ is increased while $\tilde{P}_{2}$ is decreased, which leads us to this conclusion that in general there is no assignment that minimizes all base station powers among all feasible assignments.

\section{CONCLUSION}

We have stressed the differences of joint power control and base station assignment in uplink and downlink. We have shown that there is no Pareto optimal solution for joint problem in downlink. Therefore, we have proposed an algorithm that finds a feasible solution for the joint problem when there exists any. Moreover, we have proved tht in the case that the thermal noise power is the same for all receivers, the solution is also optimal in terms of the sum of base station powers.

\section{REFERENCES}

[1] R. Yates and C. Y. Huang, "Integrated power control and base station assignment," IEEE Trans. Veh. Technol., vol. 44, pp. 638-644, Aug. 1995.

[2] R. Yates, "A framework for uplink power control in cellular radio systems,” IEEE J. Select. Areas Commun., vol. 13, p. 1341, Sept. 1995.

[3] S. V. Hanly, "An algorithm for combined cell-site selection and power control to maximize cellular spread spectrum capacity," IEEE J. Select. Areas Commun., vol. 13, pp. 1332-1340, Sept. 1995.

[4] J. Zander and M. Fordigh, "Comments on "Performance of optimum transmitter power control in cellular radio systems," IEEE Trans. Veh. Technol., vol. 43, p. 636, Aug. 1994.

[5] J. Zander, "Distributed cochannel interference control in cellular radio systems," IEEE Trans. Veh. Technol., vol. 41, pp. 305-311, Aug. 1992. 